\title{
Structure Design and Optimization of Self-standing Modular Temporary Tower of 110kV Overhead Transmission Lines
}

\author{
Hu Xiaoguang ${ }^{1, a}$, Zhang Zifu ${ }^{1, b}$, Zhu Zhanwei ${ }^{2, c}$, Zhang Xueli ${ }^{3, d}$ \\ ${ }^{1}$ China Electric Power Research Institute, Xicheng District, Beijing 100055, China \\ ${ }^{2}$ State Grid Beijing Electric Power Company, Beijing 100031, China \\ ${ }^{3}$ Beijing Electric Power Economics and Technology Institute, Beijing 100055, China \\ adkyhxg@163.com, bzf@epri.sgcc.com.cn, \\ czhuzhanwei@bj.sgcc.com.cn, ,dzhangxueli310@163.com
}

Keywords: repair tower; modularization; Temporary Tower; 110kV

\begin{abstract}
The power sector often has to transfer the transmission lines, cooperating with the municipal construction, highway, railway construction. In other case, power transmission tower fall down or wire is broken caused by natural disasters, which is a serious threat to the safe operation of power transmission lines. To improve repair efficiency and quality of overhead transmission lines in the case of lins' Transformation and disasters and incidents, this paper designed a rapidly assembled Self-standing Modular Temporary Tower, which is in light weight and is easy to be assembled, for $110 \mathrm{kV}$ transmission lines. Moreover, it also put forward an optimization plan for structure of the temporary tower. The calculation method and new structures specifically proposed by this project can meet the needs of repair and transformation works of $110 \mathrm{kV}$ transmission lines, and they are of great significance to the construction of strong and smart power network.
\end{abstract}

\section{Introduction}

With the rapid development of social economy, overhead transmission lines increasingly difficult to meet the planning requirements of the appearance of the city. The power sector often has to transfer the transmission lines, cooperating with the municipal construction, highway, railway construction. The conventional method is to remove the old tower, move a short distance in the direction of the line, or shift the direction of the horizontal line. Find a new location to build a new tower, which is the same with the old one.the new tower can make space for the road struction or other project construction, and the Temporary Tower 's schematic diagram is shown in Figure 1.

Fig.1 Schematic Diagram of the Temporary Tower

In overhead transmission line's relocation project, Temporary Tower works as Temporary equipment . when the line is completed, Temporary Tower will be dismantled. As each line's design conditions are not the same, the temporary tower is removed and difficult to have the opportunity to re-use, resulting in a great waste. To avoid the waste temporary tower and improving the efficiency of such relocation project, the design of a new type of temporary tower is imminent. 


\section{Structure Form of the 110kV Self-standing Modular Temporary Tower}

The project aims to develop a temporary tower that can be modularly assembled like building blocks. This modular temporary tower can be modularly assembled according to the voltage level(under $110 \mathrm{kV}$ ) and height. That is, with a set of modular temporary tower, to meet the different voltage levels, different call high transmission line migration needs. The new designed tower can be Modular assemblied, freely dis assemblied, re-used, with a strong general. When the line is completed, temporary tower is retrieved in storehouse and ready to re-use.The temporary tower can be rapidly installed to replace the damaged permanent tower. Usually bad weather or other natural disasters will make the transmission tower fall down, which spontaneously leads to power blackouts. Therefore, repair work is immediately required. The temporary tower will be used to substitute the collapsed permanent tower so as to restore power as soon as possible. When repair works of the permanent tower are completed, wires will be transfered from the temporary tower to the new permanent tower, and the dismantled repair tower will be kept for next use. Since the temporary tower is designed for emergency wrecking, it should be in a fine shape, a light weight and have a strong bearing capacity, and it should be able to reach the desired height and can carry large loads in different weather conditions. Thus, with a temporary tower, a timely response of unexpected power failure can be made at that very instant, and corresponding losses can be minimized.The $110 \mathrm{kV}$ transmission line is now the Commonly used voltage transmission line in China. Falling down of those towers or lines transformation usually occurs. Based on requirements, such as reutilization, quick and efficient transportation and assembly of the temporary tower, this paper designed a modularized Self-standing tower, which reduced the structural weight to the largest extent, and had an easy way to transport and assemble modular sections.

Design conditions: reference wind speed,29m/s(10m from ground); ice thickness, $10 \mathrm{~mm}$; conductor, JL/J1A-400/35, Safety factor,2.5; earth wire, JLB20-100, Safety factor,3.0; Horizontal pitch, 450m; Vertical pitch $700 \mathrm{~m}$. line angle, $40^{\circ} \sim 60^{\circ}$. Electric clearance circle is shown in Figure 2.

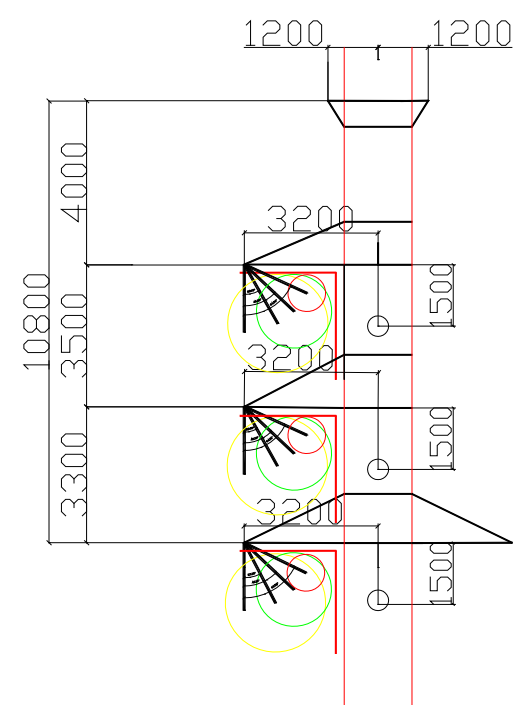

Fig.2 Electric clearance circle of the Temporary Tower

Hanging point of conductor and earth wire in tower head is shown in Figure 3. The Insulator arranged vertically in tension tower is shown in Fig.3(a), the Insulator arranged vertically in suspend tower is shown in Fig.3(b), and Insulator arranged horizontally in tension tower is shown in Fig.3(c), which is installed according to requirements of conductors. 

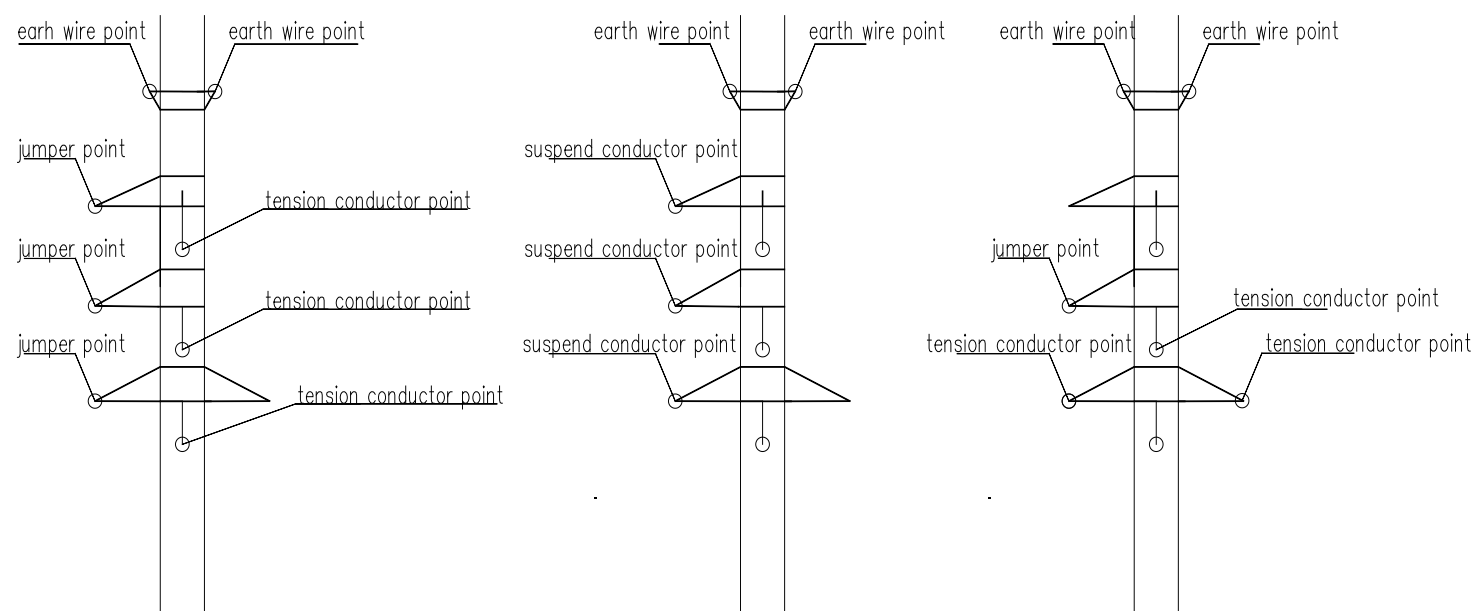

(a)Vertically in tension tower

(b)vertically in suspend tower

(c)horizontally in tension tower

Fig.3 Hanging point of conductor and earth wire in tower head

Load cases: wind case, ice case,and uneven ice case and Installation conditions, and line broken case is not consideration. There are 26 cases for suspended tower and 49 cases for tension tower. Because of different arrangement of conductor in tension tower, there are 98 cases in calculating.

\section{Structure Design and Optimization}

The modular temporary tower use Q345 steel angle, Q420 steel angle, Q345 steel pipe and Q420 steel pipe separately, and calculate the tower. Q420 steel angle and pipe are combined with the greatest intensity. The entire Tower is shown in Figure 4.

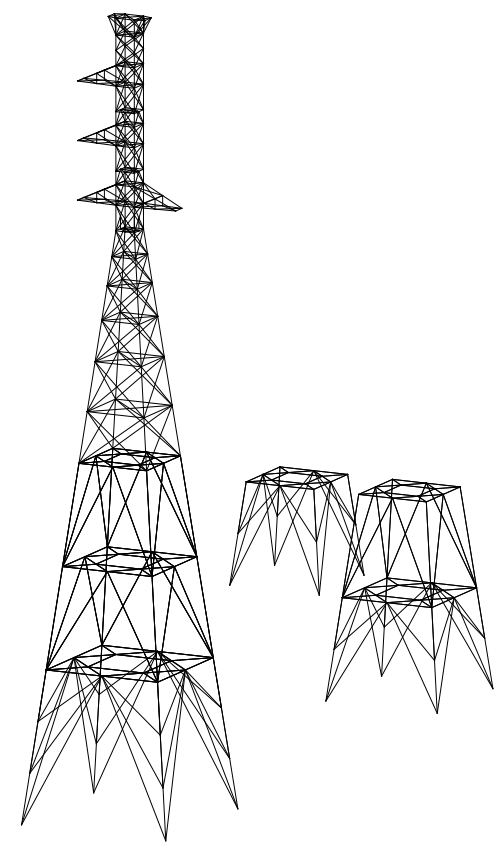

Fig.4 3Dmodal of temporary tower

The width of the tower body's top and buttom impact on the tower's overall stiffness and tower weight. The smaller the width of the tower, the smaller the rigidity of the tower head, and bring up the greater the displacement and deformation of the tower head, while the greater internal force happen in member of the head tower,resulting in increasing of tower weight. While , Increasing the width of the tower body lead to the increase in the size of the tower member, and the tower will be more and more heavy. So ,The most appropriate width is, under the condition that ensure the overall stiffness of the tower, to minimize the width, in order to take full advantage of the carrying capacity of components to achieve the lightest tower weight. 
The size of the tower body's top and buttom impact on the slope of tower's chord member and tower weight. When the width of tower body is fixed, tower's brace member become short with small slope, at the same time,internal force decrease, lowering the weight of brace member. While the internal force in chord member increase and the weight increase. Increase the tower body's slope, width of tower leg increases too, which reducing the chord member's weight and increasing the brace member weight. In addition, The width of the tower body impacts on load of basis and covered ground area. To find the suitable width of tower body is the key of optimize the tower. According to different width and slope of the tower body,tower weight were gotten, which is shown in table 1.

Table.1 optimizaiton of width and slope of tower body

\begin{tabular}{|c|c|c|c|c|c|}
\hline \multirow{2}{*}{$\begin{array}{c}\text { Width of leg } \\
(\mathrm{mm})\end{array}$} & \multirow{2}{*}{$\begin{array}{l}\text { Width of tower top } \\
\text { (mm) }\end{array}$} & \multirow{2}{*}{ slope } & \multirow{2}{*}{$\begin{array}{c}\text { Calculated weight } \\
(\mathrm{kg})\end{array}$} & \multicolumn{2}{|c|}{ Basis force $(\mathrm{kN})$} \\
\hline & & & & Pull force & Push force \\
\hline \multirow{7}{*}{9000} & 1000 & 0.232 & 15735 & 863 & -1056 \\
\hline & 1100 & 0.229 & 15724 & 864 & -1056 \\
\hline & 1200 & 0.226 & 15772 & 867 & -1057 \\
\hline & 1300 & 0.223 & 15945 & 870 & -1061 \\
\hline & 1400 & 0.220 & 15953 & 872 & -1061 \\
\hline & 1500 & 0.217 & 16105 & 875 & -1064 \\
\hline & 1600 & 0.214 & 16253 & 880 & -1068 \\
\hline \multirow{7}{*}{9200} & 1000 & 0.238 & 15803 & 844 & -1039 \\
\hline & 1100 & 0.235 & 15792 & 845 & -1039 \\
\hline & 1200 & 0.232 & 15840 & 847 & -1041 \\
\hline & 1300 & 0.229 & 15925 & 850 & -1043 \\
\hline & 1400 & 0.226 & 16021 & 852 & -1045 \\
\hline & 1500 & 0.223 & 15557 & 856 & -1044 \\
\hline & 1600 & 0.220 & 15739 & 860 & -1048 \\
\hline \multirow{7}{*}{9400} & 1000 & 0.243 & 15288 & 825 & -1021 \\
\hline & 1100 & 0.241 & 15233 & 827 & -1020 \\
\hline & 1200 & 0.238 & 15237 & 828 & -1020 \\
\hline & 1300 & 0.235 & 15410 & 831 & -1024 \\
\hline & 1400 & 0.232 & 15920 & 834 & -1028 \\
\hline & 1500 & 0.229 & 16072 & 837 & -1031 \\
\hline & 1600 & 0.226 & 16255 & 841 & -1035 \\
\hline
\end{tabular}

As can be seen from Table 1, when the slope measures 0.241 , width of top is $1100 \mathrm{~mm}$, width of leg is $9400 \mathrm{~mm}$, the tower has lightest weight,15.233t. When the slope measures 0.238 , width of top is $1200 \mathrm{~mm}$, width of leg is $9400 \mathrm{~mm}$, the tower weights, $15.237 \mathrm{t}$, almost the same with the former. While the later has better Stiffness of upper part of tower. So , modular temporary tower's important design parameters, width of tower top value $1200 \mathrm{~mm}$, width of tower leg value $9400 \mathrm{~mm}$.

\section{Summary}

On the basis of design conditions, including terrain conditions and load conditions, this paper designed a Self-standing Modular Temporary Tower of $110 \mathrm{kV}$ Overhead Transmission Lines. Findings and conclusions of this paper are as follow:

(1) A sel-stand modular temporary tower was designed for $110 \mathrm{kV}$ transmission liner;

(2) An optimizing design of the structure of the self-standing modular temporary tower was carried out;

\section{References}

[1] Mechanical Design Manual [M]. Beijing: Mechanical Industry Publishing House, 1991. 
[2] Cheng Yingtang. Design, installation, Test and Application of Hardwares of the Transmission Line [M]. Beijing: Water Conservancy and Electric Power Press, 1989.

[4] Manual of the Electric Power Fittings, Dong Ji’e, China Electric Power Press

[5] The Ultra-High Voltage Grid, Liu Zhenya (Ed.), China Economic Publishing House

[6] Electrical Power Engineering High-Voltage Transmission Line Design, Zhang Diansheng (Ed.), China Electric Power Press

[7] The Power Industry Standards of People's Republic of China. Technical Regulations of Design for Tower and Pole Structures of Overhead Transmission Line（DL/T 5154-2012）[S]. 2012. 